\title{
Secretion-Recapture Process of Apolipoprotein E in Hepatic Uptake of Chylomicron Remnants in Transgenic Mice
}

\author{
Hitoshi Shimano, Yoshio Namba, * Junichi Ohsuga, Masako Kawamura, Koji Yamamoto, Masako Shimada, \\ Takanari Gotoda, Kenji Harada, Yoshio Yazaki, and Nobuhiro Yamada \\ The Third Department of Internal Medicine and *Department of Neuropathology, Institute of Brain Research, \\ Faculty of Medicine, University of Tokyo, Tokyo 113, Japan
}

\begin{abstract}
To investigate the role of apoE in hepatic uptake of chylomicron remnants, we studied chylomicron metabolism in transgenic mice overexpressing apoE in the liver. Plasma clearance of injected ${ }^{125}$ I-labeled human chylomicrons was fivefold faster in transgenic mice than in controls. Immunohistochemistry demonstrated that apoE was specifically localized at the basolateral surface of hepatocytes from fasted transgenic mice. After injection of a large amount of chylomicrons, the density of the cell surface apoE was markedly reduced and vesicular staining was observed in the cytoplasm, suggesting that the cell surface apoE was used for hepatic endocytosis of chylomicrons and remnants. Polyacrylamide gel analysis of chylomicrons and remnants that had been reisolated from plasma and from liver membrane after the injection of chylomicrons showed the particles to be enriched with apoE mainly after their influx into the liver rather than during their residence in plasma. These results provide strong evidence for the secretion-recapture process of apoE, whereby chylomicron remnants enter the sinusoidal space, acquire apoE molecules, and subsequently are endocytosed. Data from experiments with very low density lipoprotein and LDL showed that this system is specific for chylomicron remnants. (J. Clin. Invest. 1994. 93:2215-2223.) Key words: apolipoprotein • chylomicron • lipoprotein • low density lipoprotein receptor $\bullet$ sinusoidal space
\end{abstract}

\section{Introduction}

ApoE is mainly produced in the liver and is a major constituent of mammalian lipoproteins. This protein, along with apoB100, plays a crucial role in the metabolism of plasma lipoproteins mainly through its interaction with the LDL receptor in the liver $(1,2)$. ApoE is also thought to be a specific ligand for a putative hepatic chylomicron remnant receptor and to be involved in the metabolism of intestinal lipoproteins $(1,2)$. Several lines of evidence from both in vitro and in vivo studies

Address correspondence to Nobuhiro Yamada, The Third Department of Internal Medicine, Faculty of Medicine, University of Tokyo, 7-3-1 Hongo, Bunkyo-ku, Tokyo, Japan 113.

Received for publication 7 July 1993 and in revised form 27 December 1993.

1. Abbreviations used in this paper: LRP, low density lipoprotein receptor-related protein.

J. Clin. Invest.

(c) The American Society for Clinical Investigation, Inc. $0021-9738 / 94 / 05 / 2215 / 09 \$ 2.00$

Volume 93, May 1994, 2215-2223 suggest that lipoproteins containing several molecules of apoE have higher affinity for LDL receptors than those without apoE (3-11). Recently, we established lines of transgenic mice with high plasma levels of rat apoE, which is overproduced in the liver under the control of the metallothionein promoter (12). Homozygotes for line 4-20, the highest liver expressor, exhibited a marked reduction in plasma cholesterol and triglyceride levels, with elimination of VLDL and a marked decrease in LDL (13). These decreases were caused by enhanced plasma clearance of lipoproteins containing apoB, which were enriched with apoE (13). Kinetic studies of plasma lipoproteins in these animals demonstrated that hepatic overexpression of apoE markedly enhances plasma turnover of VLDL and LDL through interactions with LDL receptors and possibly with LDL receptor-related proteins $(\mathrm{LRP})^{1}(14)$. We also performed oral challenge with retinyl palmitate as a marker for chylomicron remnant metabolism, and the data suggested that hepatic overexpression of apoE markedly enhances clearance of chylomicron remnants (14). In contrast with VLDL, which is produced in the liver, chylomicron particles are produced and secreted by the intestine. In our transgenic line, apoE is expressed in the liver and not in the intestine. The enhanced clearance of chylomicron remnants in hepatic overexpression of apoE means that chylomicron particles must obtain the apoE molecules at some point in the pathway through which they are metabolized in plasma, finally to be taken up by the liver.

In the study reported here, we directly measured plasma clearance of ${ }^{125} \mathrm{I}$-labeled human chylomicrons and compared the apoE content of chylomicrons and remnants reisolated from plasma and liver after injection of chylomicrons. For further study of the mechanism by which hepatic overexpression of apoE enhances hepatic uptake of chylomicrons, distribution of apoE in hepatocytes and changes in its distribution after injection of lipoproteins were investigated by immunohistochemistry. The data suggested that chylomicrons and their remnants were enriched with apoE molecules on the surface of hepatocyte in the sinusoidal space and were immediately endocytosed by hepatic receptors. This local apoE shuttle mechanism was specific for chylomicron remnants and not for hepatic lipoproteins.

\section{Methods}

Materials. $\mathrm{Na}^{125}{ }^{125}$ and ${ }^{125}$ I-Protein A were purchased from NEN-Du Pont (Boston, MA). BSA, PMSF, and Triton X-100 were obtained from Sigma Chemical Co. (St. Louis, MO). All reagents used were of analytical grade. BDF1 mice were from Nippon Bio-Supp. Inc. (Tokyo, Japan).

Animals. Transgenic mice, MAE 4-20, which overexpress rat apoE under the control of the metallothionein promotor, MAE 4-20 were established as described previously $(12,13)$. Homozygous and hetero- 
zygous line 4-20 aged 16-20 wk were used in this study. Normal mice with the same genetic background ([C57BL6 $\times$ BDA] F2 hybrids) and of the same age were used as controls for MAE 4-20. The animals were maintained on normal chow and given water supplemented with 20 $\mathrm{mM} \mathrm{ZnSO}_{4}$ for $>1 \mathrm{wk}$ to induce the expression of rat apoE from the transgene. Before intravenous injection of lipoproteins for kinetic studies and immunohistochemistry, the animals were fasted for $12 \mathrm{~h}$.

Lipoproteins and iodination. Chylomicrons were prepared from pleural effusion of a patient with malignant lymphoma and chylothorax. VLDL and LDL were prepared from plasma drawn from normolipidemic volunteers. VLDL from a patient with apoE deficiency was kindly donated by Dr. T. Teramoto (Teikyo University, Tokyo, Japan). Fresh effusion and plasma samples were separated with disodium EDTA $(1 \mathrm{mg} / \mathrm{ml})$, sodium azide $(0.2 \mathrm{mg} / \mathrm{ml})$, and benzamidine $(300 \mu \mathrm{g} / \mathrm{ml})$.

Chylomicrons were separated by ultracentrifugation at $40,000 \mathrm{rpm}$ in a rotor (SW28; Beckman Instruments, Inc., Fullerton, CA) for $0.5 \mathrm{~h}$, followed by reultracentrifugation at $20,000 \mathrm{rpm}$ for $16 \mathrm{~h}$. The composition was (wt/wt \%): total protein $4.8 \%$, triglycerides $77 \%$, phospholipids $6.7 \%$, free cholesterol $3.0 \%$, and cholesterol ester $8.5 \%$. For separation of VLDL and LDL, plasma was ultracentrifuged in a 50.2-Ti rotor at $45,000 \mathrm{rpm}$ at $12^{\circ} \mathrm{C}$ at $\mathrm{KBr}$ densities of 1.006 and 1.063 for 16 and $20 \mathrm{~h}$, respectively (15). The floating lipoproteins were reultracentrifuged in a 40.3 rotor and dialyzed against $150 \mathrm{mM} \mathrm{NaCl}, 2 \mathrm{mM}$ sodium phosphate ( $\mathrm{pH} 7.4$ ), $0.1 \mathrm{mg} / \mathrm{ml}$ EDTA, and $0.2 \mathrm{mg} / \mathrm{ml} \mathrm{so-}$ dium azide. Chylomicrons and VLDL were iodinated with ${ }^{125} \mathrm{I}$ by the method of McFarlane (16) with slight modification as previously described (14).

Kinetic studies. Turnover studies of iodinated chylomicrons and VLDL were performed as previously described (14). For calculation of chylomicron kinetics, the injected apoB count of tracer per estimated plasma volume ( $4.5 \%$ of body wt) was taken as $100 \%$. For VLDL kinetics, the radioiodine in apoB in plasma prepared $1 \mathrm{~min}$ after the injection was taken as $100 \%$ at time 0 .

Immunohistochemical procedures for light microscopy. Animals fasted for $12 \mathrm{~h}$ were anesthetized with pentobarbital. Lipoproteins $(0.3$ $\mathrm{mg}$ protein of chylomicrons or VLDL or $0.5 \mathrm{mg}$ protein of LDL/ $100 \mu$ PBS) or saline as a control were injected through the tail vein. $7 \mathrm{~min}$ after injection, the livers were perfused with ice-cold saline through a portal vein and resected. Small aliquots from the right lobes of the resected livers were immediately fixed in $4 \%$ paraformaldehyde in PBS (pH. 7.4). Other portions of the samples were used for preparation of membrane pellets. Paraformaldehyde-fixed, paraffin-embedded sections were subjected to immunohistochemistry (17). For apoE immunostaining, two primary antibodies were used: a rabbit anti-rat apoE polyclonal antibody, which detects both rat and mouse apoE's, and rabbit polyclonal antibody to rat apoE-specific synthetic oligopeptide, which does not cross-react with mouse apoE (12). Antiserum was diluted to 1:200 with PBS. The sections of livers were incubated with the respective antibodies overnight. After washing with PBS, the biotinylated second antibodies (goat anti-rabbit IgG antibody) were added. The sections were visualized by the addition of peroxidase-conjugated streptavidin.

Analysis of chylomicron apoE after intravenous injection. Chylomicrons $(0.3 \mathrm{mg}$ protein) were injected into the tail vein of 12 -h fasted animals. 10 min after injection, blood was drawn by cardiac puncture. The plasma samples were layered by saline containing $0.01 \%$ EDTA, and chylomicrons and remnants were reisolated by ultracentrifugation in SW41 at 20,000 rpm for $1 \mathrm{~h}$. The livers were resected after perfusion with ice-cold saline through the portal vein and liver membrane fractions $(8000-100,000 \mathrm{~g}$ pellets) were prepared as previously described $(11,14)$. Membrane pellets were frozen at $-80^{\circ} \mathrm{C}$ and thawed in PBS containing $10 \mathrm{mM}$ Suramin. The samples were layered by saline containing $0.01 \%$ EDTA and ultracentrifuged at $20,000 \mathrm{rpm}$ for $1 \mathrm{~h}$. The floating lipoproteins were isolated and subjected to SDS-PAGE.

Electrophoresis and immunoblot. The apoE in chylomicron samples was separated by polyacrylamide gel electrophoresis in the presence of SDS as described by Laemmli (18). Aliquots of the chylomi- crons containing $100 \mu \mathrm{g}$ of triglycerides were dissolved in $50 \mu \mathrm{l}$ of $3 \%$ SDS, $62.5 \mathrm{mM}$ Tris- $\mathrm{HCl}$ ( $\mathrm{pH} 6.8$ ), $10 \%$ glycerol, $0.025 \%$ bromophenol blue, and $5 \%$ mercaptoethanol, and heated at $100^{\circ} \mathrm{C}$ for $3 \mathrm{~min}$. Electrophoresis was performed in $11 \%$ polyacrylamide slab gels containing $1 \%$ SDS. After electrophoresis, the gel was stained with Coomassie brilliant blue. In some experiments, the proteins were electrophoretically transferred to nitrocellulose membranes in the glycine buffer at $200 \mathrm{~mA}$ for $8 \mathrm{~h}$. The sheet was incubated with the same buffer containing anti-rat apoE serum (11) for $12 \mathrm{~h}$ at $4^{\circ} \mathrm{C}$, followed by incubation in the buffer containing ${ }^{125} \mathrm{I}$-Protein A $(2,000 \mathrm{cpm} / \mathrm{ng}, 0.5 \mu \mathrm{g} / \mathrm{ml})$ for $2 \mathrm{~h}$ at room temperature. The sheets were washed, air dried, and autoradiographed on x-ray film.

\section{Results}

Kinetics of ${ }^{125}$ I-chylomicrons and ${ }^{125} I-V L D L .{ }^{125}$ I-labeled chylomicrons were injected intravenously, and plasma clearances were compared in transgenic mice and control (Fig. 1). Since the values were calculated from the radioactivities in apoB in ${ }^{125}$ I-labeled chylomicrons, the data represented plasma clearance of in terms of the number of particles. The injected chylomicrons were much more rapidly cleared in transgenic mice than in controls. In the first $5 \mathrm{~min}, 59$ and $22 \%$ of apoB in injected chylomicrons were removed in transgenic and control mice, respectively. In one experiment, livers were resected 10 min after the injection and the total counts in the liver were measured after extensive perfusion. The total count in the liver of transgenic mice was $72 \%$ of total injected count and was threefold higher than in control $(n=2)$. Thus, injected chylomicrons were taken up primarily by the liver and the rapid plasma clearance in transgenic mice was attributed mainly to enhanced hepatic uptake. In contrast, plasma clearance of ${ }^{125} I-$ labeled human VLDL was similar in the transgenic and control mice (Fig. $2 A$ ). This was in striking contrast to our previous finding that plasma clearance of VLDL prepared from transgenic mice, and thus already apoE-rich, was markedly faster in

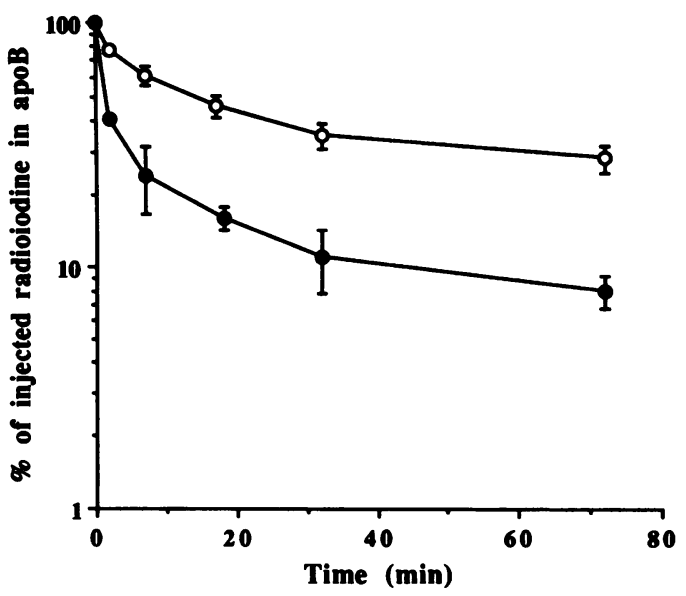

Figure 1. Plasma turnover of ${ }^{125} \mathrm{I}$-labeled human chylomicrons in apoE transgenic mice. ${ }^{125} \mathrm{I}$-labeled human chylomicrons $(1 \mu \mathrm{Ci} / 100$ $\mu \mathrm{l}$ per animal) was injected into transgenic mice (homozygous MAE 4-20) $(\bullet)$ and controls $(O)$ through the tail vein $(n=3)$. At designated intervals after injection, blood samples were drawn from the retroorbital vein for determination of plasma radioactivity in apoB by the isopropanol precipitation method. The increase in the plasma triglyceride level of any sample at $2 \mathrm{~min}$ after the injection did not reach $5 \mathrm{mg} / \mathrm{dl}$ in any sample. Values are percentages of the injected radioiodine in apoB remaining in the plasma. Each point represents the mean \pm SD (bar). 
$\mathbf{A}$

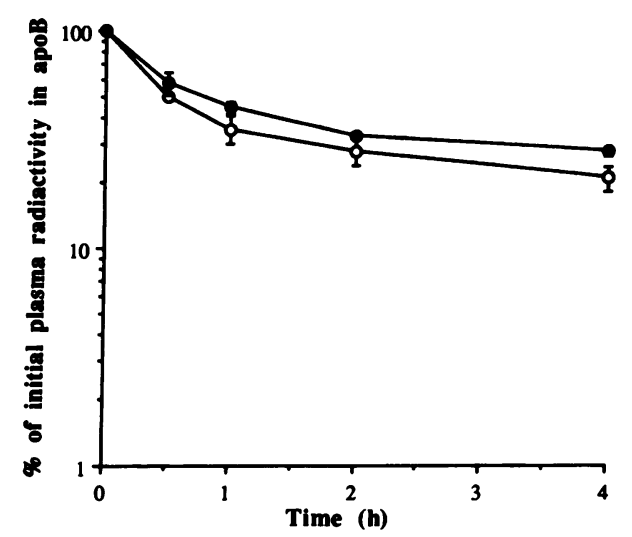

B

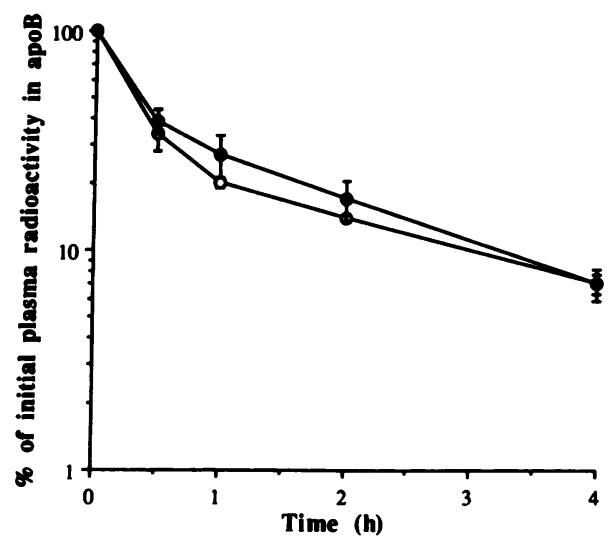

Figure 2. Plasma turnover of ${ }^{125} \mathrm{I}$-labeled human VLDL $(A)$ and apoE-deficient VLDL $(B)$ in apoE transgenic mice. Human VLDL and apoE-deficient VLDL were prepared from a normolipidemic volunteer with the E3/E3 isoform and a patient with apoE deficiency, respectively. Each VLDL sample was labeled with ${ }^{125}$ I and injected $(1 \mu \mathrm{Ci} / 100 \mu \mathrm{l}$ per animal) into transgenic mice (homozygous MAE 4-20) $(\bullet)$ and controls $(O)$ through the tail vein $(n=3)$. At designated intervals after injection, blood samples were drawn from the retroorbital vein for determination of plasma radioactivity in apoB by the isopropanol precipitation method. Blood samples obtained $1 \mathrm{~min}$ after injection were taken as time 0 . The increase in plasma triglyceride level was not significant in any sample. Values are percentages of time 0 plasma radioactivity in apoB remaining in blood plasma. Each point represents the mean $\pm \mathrm{SD}$ (bar).

transgenic mice than that of endogenous VLDL in control mice (14). It is possible that the human VLDL used was sufficiently apoE rich, and that there was no space for additional incorporation of rat apoE to enhance plasma clearance. To exclude this possibility, we performed a kinetic study of VLDL in the presence of apoE deficiency. The removal of ${ }^{125} \mathrm{I}-\mathrm{VLDL}$ prepared from apoE-deficient patients was also similar between the two groups (Fig. $2 B$ ). These data indicated that hepatic overexpression of and high plasma level of apoE had no effect on plasma clearance of exogenous human VLDL.

Immunohistochemical localization of apoE in liver of transgenic mice. For determination of the direct involvement of hepatic overexpression of apoE in enhanced chylomicron remnant clearance, livers of transgenic mice were immunohistochemically studied with rat apoE-specific antibody. This antibody detects only the transgene product, rat apoE, and not mouse or human apoE (13). As shown in Fig. $3 A$, marked immunostaining of apoE was seen at the sinusoidal front in transgenic mouse liver. The distribution was uniform across the hepatic lobule, from the portal tracts to the central veins. At higher magnification (Fig. $3 B$ ), apoE was found peripherally near the basolateral surface of every hepatocyte, while minimal in the cytoplasm. This result was consistent with apoE localization in the liver of rat as previously reported (19). There was no marked basolateral staining in the liver of control animal (Fig. $3 C$ ). When control liver was stained with rat apoE polyclonal antibody cross-reactive with mouse apoE, a similar sinusoidal pattern was found in hepatocytes of control animals, although it was less intense than in transgenic mice (Fig. 3, $D$ and $E$ ), indicating that the transgene product and endogenous mouse apoE were distributed similarly. As a test of whether or not the basolateral hepatic apoE was used for hepatic uptake of chylomicron remnants, a large amount of chylomicron $(0.3 \mathrm{mg}$ protein) was injected intravenously in a bolus and the changes in apoE distribution were studied. $7 \mathrm{~min}$ after the injection of chylomicrons, the liver was resected and stained. This was the interval during which $75 \%$ of labeled chylomicron was cleared in the transgenic mice, and thus was estimated to be long enough for a large quantity of injected chylomicron to be taken up. Sinusoidal immunostaining of apoE was markedly reduced as compared to the preinjection level (Fig. 4, $A$ and $B$ ). Instead, immunostaining was observed in a vesicular pattern in the cytoplasm. This change in the immunostaining pattern suggests that in the process of hepatic uptake of large amounts of injected chylomicrons, basolateral apoE was consumed and reduced, while endosomes containing apoE-rich lipoproteins appeared in a vesicular pattern in the cytoplasm. In contrast, injection of VLDL and LDL had no effect on the sinusoidal staining of apoE, although slight vesicular staining appeared in the cytoplasm (Fig. 5, $A$ and $B$ ).

Estimation of apoE content of chylomicrons and remnants after intravenous injection of chylomicrons. Enhanced clearance of chylomicron remnants in transgenic mice may be caused by an increased number of apoE molecule on the particles, which would increase their affinity for hepatic receptors. To estimate the extent of transfer of apoE to injected chylomicron particles, chylomicron remnants were reisolated from plasma and liver membrane after injection of chylomicrons, and subjected to SDS polyacrylamide gel analysis (Fig. 6). Since the amount of injected chylomicrons would be in excess of the capacity of lipoprotein lipase in the mouse, these reisolated particles probably contained much unhydrolyzed chylomicrons, as well as their remnants. Human apoE was observed in original human chylomicrons used for injection at an apparent molecular mass of $35 \mathrm{kD}$ in doublets, resulting from the presence of its sialylated form. As shown in Fig. $6 \mathrm{~A}$, chylomicrons and remnants reisolated from plasma of transgenic mice had a trace amount of human apoE and a small amount of rat apoE, the transgene product of $34 \mathrm{kD}$. In contrast, when prepared from the liver membrane fraction after injection, chylomicrons and remnants from transgenic mice were highly enriched with rat apoE and contained a markedly higher level of apoE than the original particles (Fig. $6 \mathrm{~B}$ ). These data were confirmed by immunoblot using rat apoE-specific antibody (Fig. 7). In transgenic mice, the particles reisolated from liver membrane were estimated to be fourfold enriched with rat apoE as compared to those from plasma. As compared to the injected particles, the contents of apoC's were grossly increased in the chylomicrons in plasma and markedly reduced in liver 

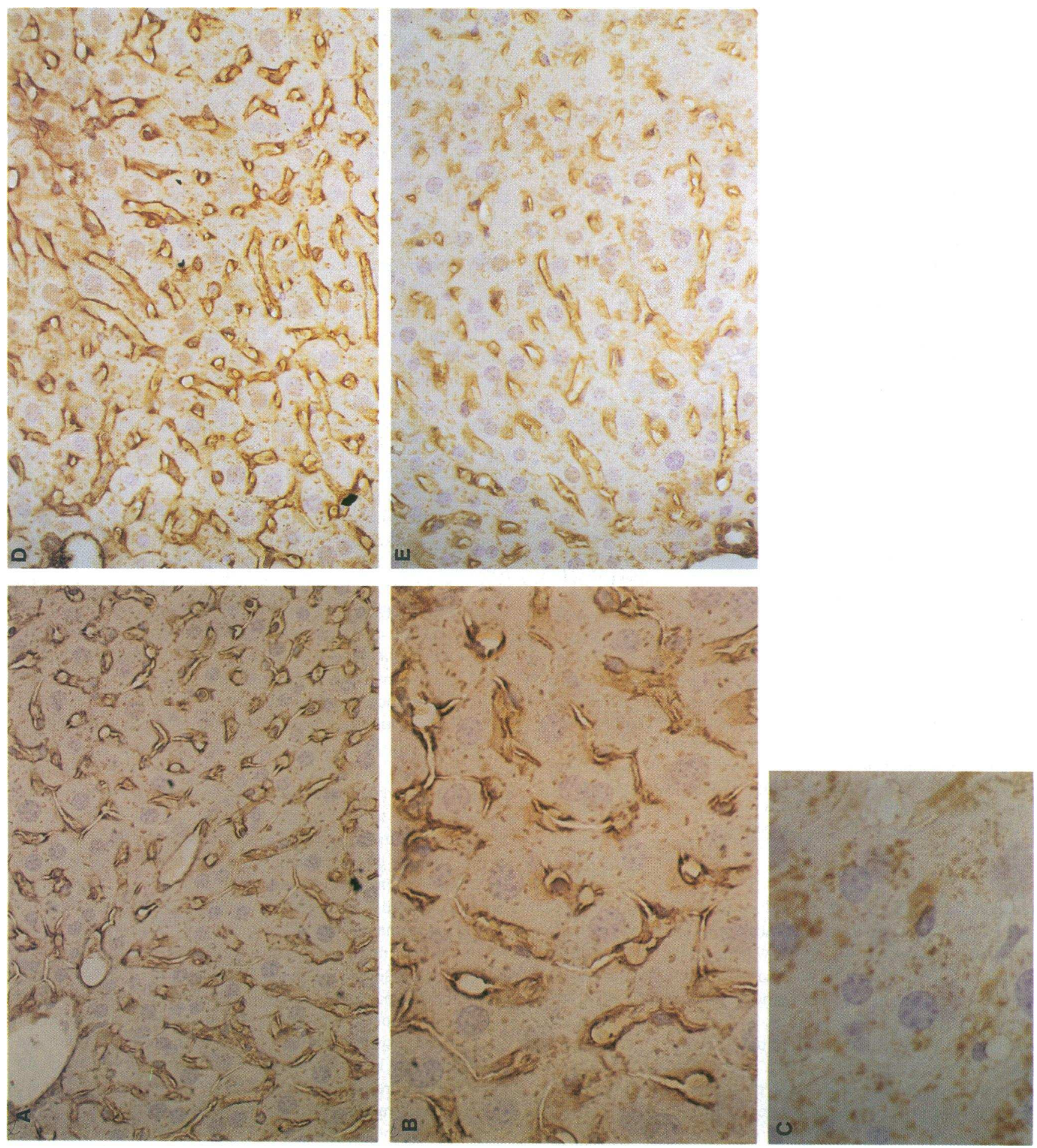

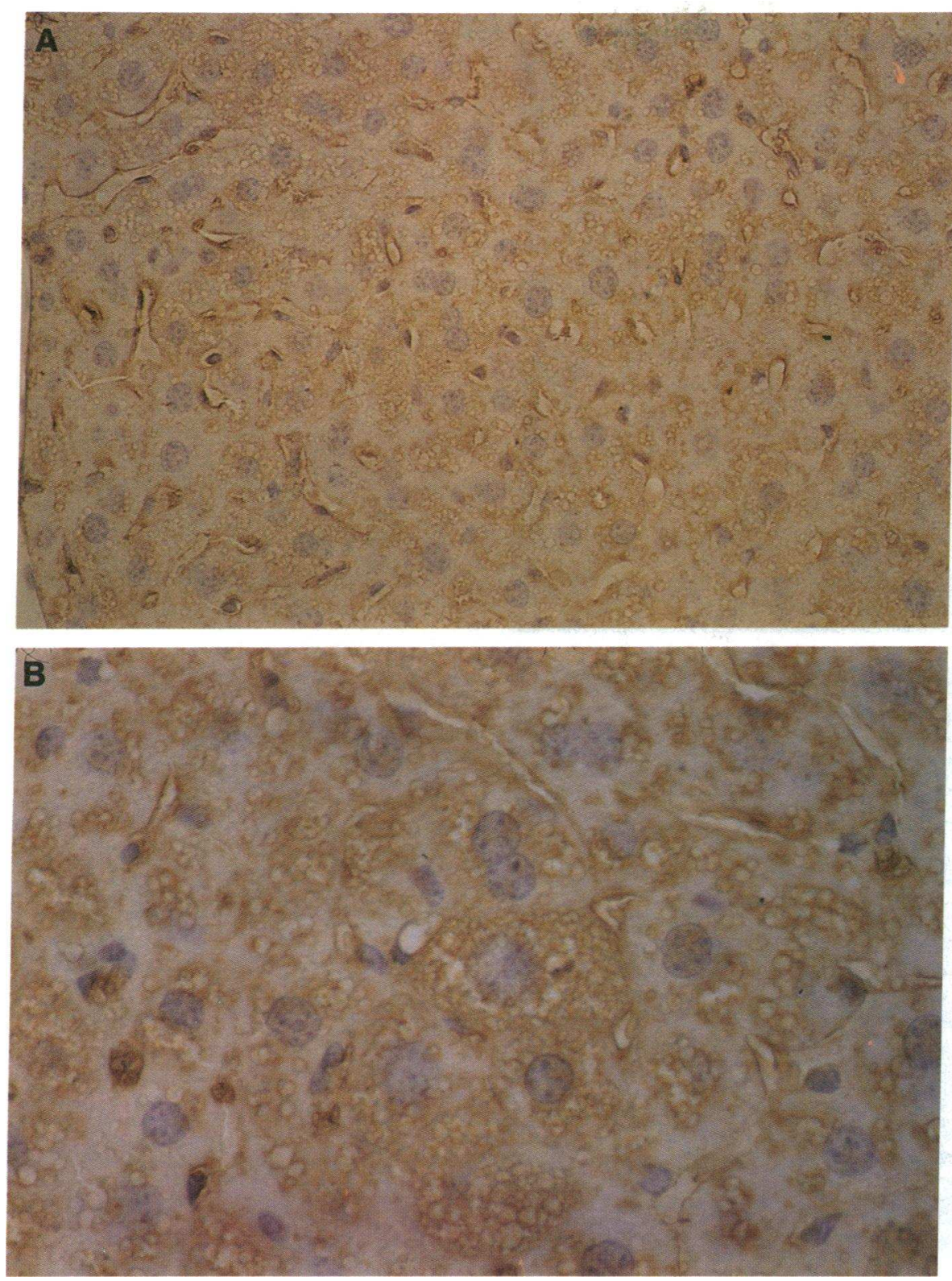

Figure 4. Changes in hepatic localization of apoE after injection of chylomicrons as assessed by immunohistochemistry. Human chylomicrons ( $0.3 \mathrm{mg}$ protein/ $100 \mu \mathrm{l}$ PBS) were injected into transgenic mice (homozygous MAE 4-20) through the tail vein. 7 min later, blood was drawn by cardiac puncture, and the liver was perfused with ice-cold saline and resected for immunohistochemistry with anti-rat apoE-specific antibody. The plasma triglyceride level in blood samples was 300 $400 \mathrm{mg} / \mathrm{dl}$. Representative data from several experiments are shown. $A, \times 50$; $B, \times 100$. membranes (Fig. 6). This suggests that mouse apoC's might be considerably transferred to chylomicrons and remnants, displace human apoE in plasma, and then be replaced by rat apoE in the liver. These data suggest that the transfer of apoE to chylomicrons and remnants occurred mainly after their influx into the liver rather than while they were in the circulation. There may be some methodological problems in this experiment. First, chylomicrons and remnants that were reisolated from plasma $10 \mathrm{~min}$ after injection may not reflect the particles that had been already cleared, especially for apoE content. In some experiments, blood was drawn after different intervals such as $1 \mathrm{~min}$ and $5 \mathrm{~min}$ after the injection. These reisolated particles contain apoE in amounts different, but consistently much smaller than the particles respectively reisolated from liver membrane. Second, apoE-rich VLDL produced in the liver may contaminate the particles reisolated from liver membrane and cause overestimation of apoE content on remnants reisolated from liver membrane, although the condition of ultracentrifugation was for chylomicrons and not for VLDL. When prepared from liver membrane without injection of chylomicrons, total amount of refloated lipoproteins, which were considered to be mainly newly synthesized VLDL, was $<5 \%$ (in triglycerides) that of lipoproteins reisolated from liver membrane after the injection, suggesting that reisolated lipoprotein after the injection was mainly chylomicrons and remnants. Taken together with the presence of apoC's in plasma as

Figure 3. Immunohistochemical localization of apoE in liver of transgenic $(A, B$, and $D)$ and control mice $(C$ and $E)$. Liver samples from transgenic (homozygous MAE 4-20) and control mice were processed for immunostaining with two polyclonal anti-rat apoE antibodies. The antibody to purified whole rat apoE, used in $D$ and $E$, reacts with both rat and mouse apoE. The antibody to rat apoE-specific oligopeptide, used in $A-C$, detects only rat apoE. The specificities of these antibodies were previously confirmed by immunoblot analysis of rat and mouse plasma samples (12). $A, D$, and $E, \times 50 ; B$ and $C, \times 100$. 

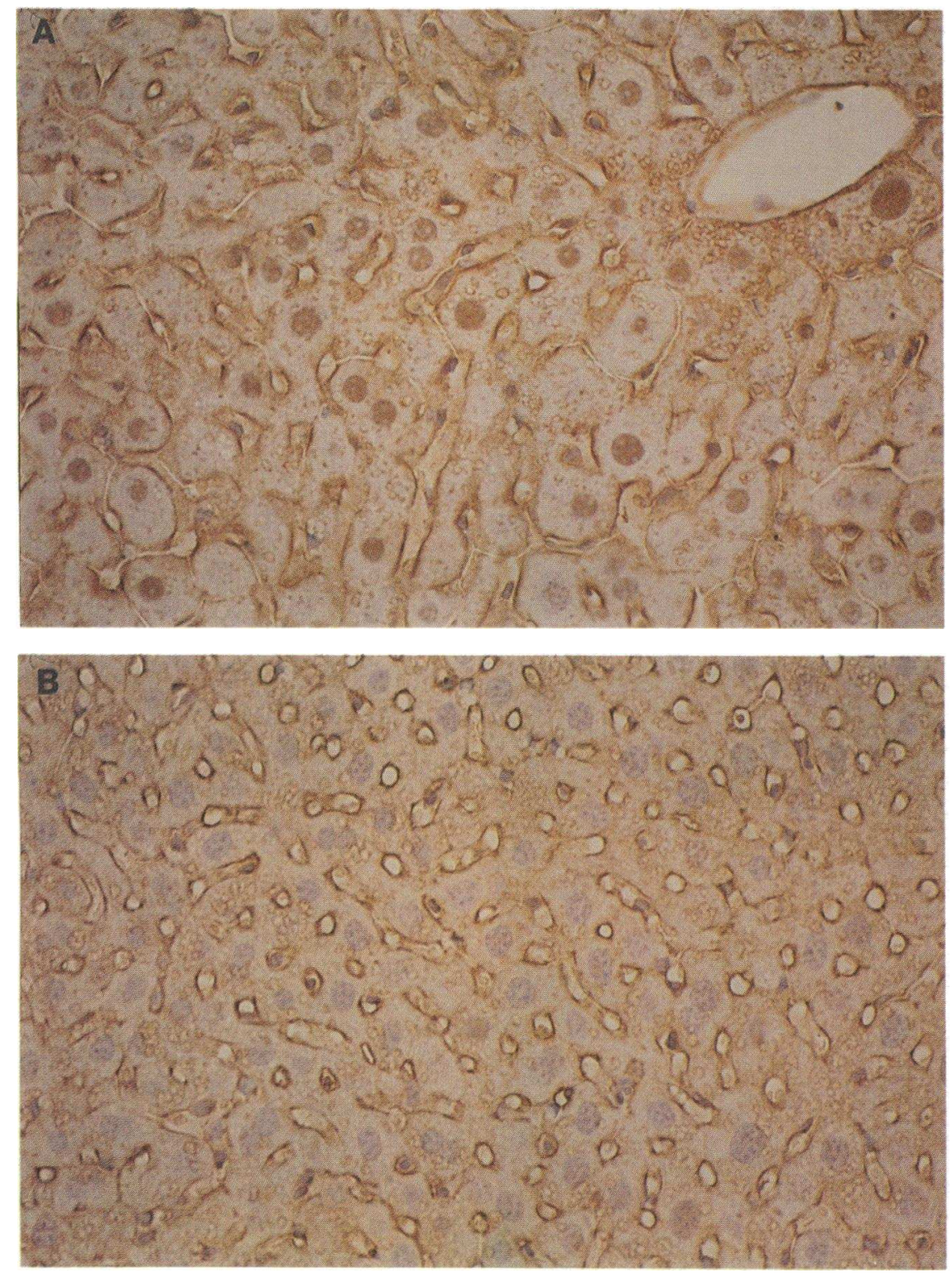

Figure 5. Changes in hepatic localization of apoE after injection of VLDL $(A)$ and LDL $(B)$ as determined by immunohistochemistry. Human VLDL and LDL from a normolipidemic volunteer with the apoE3 isoform $(0.5 \mathrm{mg}$ protein $/ 100 \mu \mathrm{PBS})$ were injected into transgenic mice (homozygous MAE 4-20) through the tail vein. 7 min later, blood was drawn by cardiac puncture and the liver was perfused with ice-cold saline and resected for immunohistochemistry with anti-rat apoE-specific antibody. $\times 50$.

a competitor for plasma transfer to chylomicrons and remnants, the main site of apoE transfer to the particles was considered to be the sinusoidal space.

\section{Discussion}

The data from this kinetic study of ${ }^{125}$ I-chylomicrons are highly consistent with the results of our previous study (14), in which we used oral retinyl palmitate loading as a marker for chylomicron remnant metabolism: in that investigation, plasma levels of retinyl palmitate after oral administration were fivefold lower in transgenic mice than in controls. It is now established that hepatic overexpression of apoE markedly enhances plasma clearance of chylomicron remnants. A high apoE content on chylomicron remnants would be essential to an effi- cient receptor mediated-endocytosis, although functional proposition of LDL receptors and chylomicron remnant receptors that might be LRP is controversial (19-22). The chylomicrons used in this study were prepared from pleural effusions of a patient with chylothorax and were relatively poor in apoE. The calculated molar ratio of apoE to apoB estimated by turbidity immunoassay was $\sim 1.3$, meaning that, on average, one particle contains only one apoE molecule. The greater part of these apoE-poor chylomicron particles have low or no affinity for the hepatic lipoprotein receptors, such as LDL receptors. However, plasma clearance of these particles was markedly enhanced in the transgenic mice, while the plasma clearance of ${ }^{125}$ I-labeled VLDL was not enhanced. The mechanism of apoE transfer might differ among lipoprotein classes. To gain the affinity for hepatic receptor, the injected chylomicron particles 


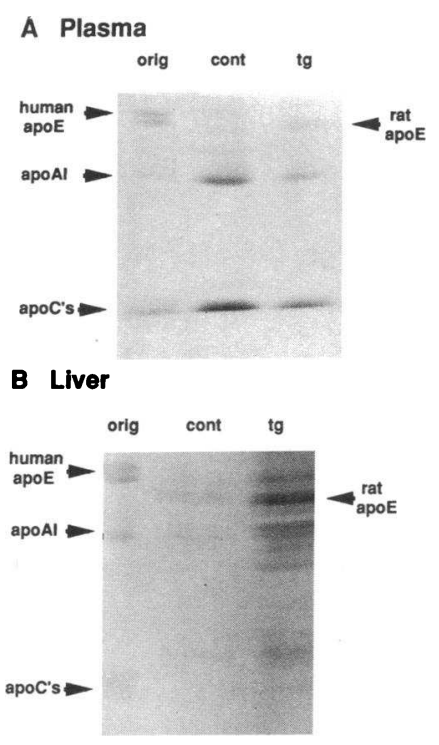

Figure 6. SDS-polyacrylamide gel analysis of apoE in chylomicrons and remnants reisolated from plasma and liver after injection of chylomicrons. Human chylomicrons were injected into transgenic and control animals. 10 min after injection, blood samples were drawn by cardiac puncture. The liver was perfused with ice-cold saline and resected for preparation of liver membrane pellets. Chylomicrons and remnants were reisolated from plasma $(A)$ and liver membrane $(B)$ samples, as described in the Methods. Each sample ( $100 \mu \mathrm{g}$ of triglyceride) from transgenic $(\mathrm{tg})$ and control ( cont $)$ mice and original

chylomicrons (ori) used for the injection were subjected to $11 \%$ SDS-PAGE and stained with Coomassie brilliant blue. Human apoE and rat apoE in doublets (in unsialylated and sialylated forms) were detected at apparent molecular mass of 35 and $34 \mathrm{kD}$, respectively, which were estimated between markers of 42.7 and $31.0 \mathrm{kD}$.

must have incorporated rat apoE molecules, which had been overproduced in the liver, before they were endocytosed. A key question is whether apoE molecules overexpressed by the liver were transferred to chylomicron remnants from other apoErich lipoproteins in the plasma or directly from the liver. To address this question, hepatic localization of apoE should be investigated.

It was reported that rat apoE molecules are localized at the basolateral surface of rat hepatocytes on the microvillous extensions into the space of Disse (19). Our present immunohistochemical data from both transgenic and control mice were consistent with this. We also confirmed the presence of apoE in the liver plasma membrane fraction by immunoblotting using apoE antibody (data not shown). The reduction in basolateral

liver plasma

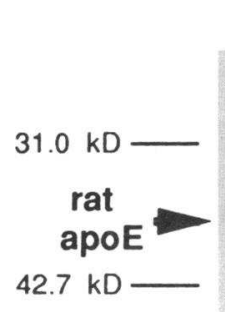

Figure 7. Immunoblot analysis of apoE in chylomicrons and remnants reisolated from plasma and liver after injection of chylomicrons. Chylomicrons and remnants were reisolated from plasma (plasma) and liver membrane (liver) samples of transgenic mice, as described in Fig. 6. Each sample ( $10 \mu \mathrm{g}$ of triglyceride) was subjected to immunoblot using rat apoE-specific antibody. Molecular weight markers are indicated.
apoE density after injection of chylomicron strongly suggests that hepatic apoE molecules aggregating at the sinusoidal front of hepatocytes were consumed for apoE enrichment of chylomicrons and remnants. Hepatic LDL receptors were reported to be situated at the basolateral surface of hepatocytes (23). The similar pattern of apoE and LDL receptors suggests that enrichment of chylomicrons and remnants with apoE may be immediately followed by hepatic uptake through LDL receptors, since several lines of evidence indicate that LDL receptors play a major role in plasma clearance of chylomicron remnants in rodents $(21,22,24)$. LRP, which is as likely a candidate as chylomicron remnant receptors, appears to be also similarly localized and involved in hepatic uptake of chylomicrons and remnants.

SDS-PAGE analysis of reisolated chylomicrons and remnants after injection of chylomicrons supports that apoE enrichment may occur mainly in the sinusoidal space and partially in the plasma. ApoC's may be involved in the limited plasma transfer of apoE to chylomicrons and remnants. ApoC's are known to inhibit the interaction of apoE with both LDL receptor (25) and LRP (26). The competitive transfer of apoE and apoC's might have resulted in a small but considerable amount of rat apoE detected in chylomicrons and remnants reisolated from plasma of transgenic mice and the almost undetectable amount of mouse apoE in plasma of control mice. Considerable plasma apoE transfer to chylomicrons and remnants may occur only in the transgenic mice with a marked high plasma apoE level. It has been reported that both apoE and apoC's were transferred to lymph chylomicrons upon incubation with plasma in rats (27).

These results, even if under unphysiological conditions, might imply an interesting mechanism of transport of intestinally absorbed lipids to the liver, presented schematically in Fig. 8 , which has been proposed first by Hamilton et al. (19) and later enunciated by others as secretion-recapture theory (28). Daily food intake results in episodic appearance of intestinal lipoproteins in the circulation. These intestinal chylomicron particles are subjected to lipolysis by lipoprotein lipase (LPL) and probably to competitive transfer of apoE and apoC's from other apoE- and apoC-rich lipoproteins. Meanwhile, apoE is produced continuously in the liver. The apoE molecules, that escaped incorporation onto nascent VLDL, are stored at the sinusoidal front of hepatocytes, ready for the influx of chylomicron remnants. Postprandially, chylomicron remnants flowing through the portal vein encounter abundant apoE molecules on the cell surface of hepatocytes or newly secreted apoE molecules in the sinusoidal space and become highly apoE-rich. It is also possible that apoE transfer may occur from apoE-rich lipoproteins (mainly HDL) to the remnants during their residence in this space. These events may be associated with replacement of apoC's on the particles in the competitive way. Subsequently, these apoE-rich particles are endocytosed through the interaction of apoE with hepatic lipoprotein receptors, which completes secretion-recapture process. If the initial capture of the remnant particles were mainly by the cell surface apoE, the rapid plasma clearance of chylomicron remnants are can be well explained by this initial sequestration in the sinusoidal space. This system would be very suitable for the removal of steep episodic and steep influx of intestinal lipoproteins, which lack apoE, into plasma. The system is specific for chylomicron remnants and not for hepatic lipoproteins such as VLDL and LDL. 


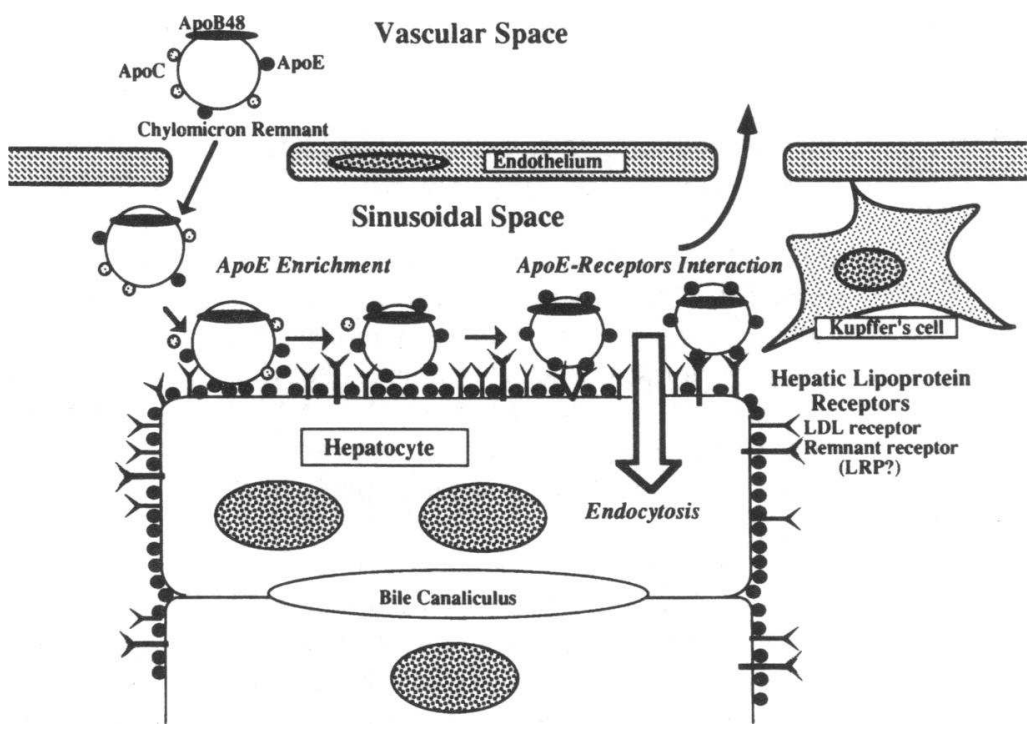

Figure 8. Schematic representation of the secretionrecapture process of apoE for hepatic uptake of chylomicrons and remnants. The means of attachment of apoE molecules to the cell surface of hepatocytes and their positional relationship to hepatic lipoprotein receptors are unknown.
How apoE molecules attach to the cell surface is unknown. They may bind to hepatic receptors as free apolipoproteins (19). Alternatively, apoE molecules may be attached to cell surface heparan sulfate proteoglycans $(19,28)$, since apoE avidly binds to proteoglycan (29) and the site of heparan sulfate of hepatocytes is similar to those of apoE and LDL receptor (30). Recent reports suggest that cell surface heparan sulfate proteoglycans function in cellular uptake of lipoproteins in vitro $(31,32)$ and initial sequestration of chylomicron remnants in sinusoidal space from plasma (33). LPL and LRP have been also proposed to be involved in this process $(32,34)$. The precise mechanism for uptake of remnants by hepatocytes involving apoE, proteoglycans, LPL, LRP, and LDL receptor is yet to be elucidated.

In the current study, it was demonstrated that hepatic overproduction of apoE enhances plasma clearance of chylomicron remnants, and that hepatic cell surface apoE might play an important role in the metabolism of these lipoproteins by the secretion-recapture mechanism. The theory proposed here is based on rat apoE, the transgene product from the liver of transgenic mice. The similar distribution of endogenous mouse apoE in the liver and a high homology between the two molecules suggest that this theory can be applied to normal mice and probably to humans.

\section{Ackowledgments}

This work was supported by a grant for diabetes research from Otsuka Pharmaceutical Co., Ltd (Tokushima, Japan).

\section{References}

1. Mahley, R. W. 1988. Apolipoprotein E: cholesterol transport protein with expanding role in cell biology. Science (Wash. DC). 240:622-630.

2. Brown, M. S., and J. L. Goldstein. 1986. A receptor pathway for cholesterol homeostasis. Science (Wash. DC). 232:34-37.

3. Pitas, R. E., T. L. Innerarity, K. S. Arnold, and R. W. Mahley. 1979. Rate and equilibrium constants for binding of apo-E HDLc (a cholesterol-induced lipoprotein ) and low density lipoproteins to human fibroblasts: evidence for multiple receptor binding of apo-E HDLc. Proc. Natl. Acad. Sci. USA. 76:2311-2315.

4. Yamada, N., D. M. Shames, J. B. Stoudmire, and R. J. Havel. 1986. Metabolism of lipoproteins containing apolipoprotein B-100 in blood plasma of rabbits: heterogeneity related to the presence of apolipoprotein E. Proc. Natl. Acad. Sci. USA. 83:3479-3483.
5. Yamada, N., D. M. Shames, and R. J. Havel. 1987. Effect of low density lipoprotein receptor deficiency on the metabolism of apolipoprotein B-100 in blood plasma. J. Clin. Invest. 80:507-515.

6. Yamada, N., D. M. Shames, K. Takahashi, and R. J. Havel. 1988. Metabolism of apolipoprotein B-100 in large very low density lipoproteins of blood plasma. J. Clin. Invest. 82:2106-2113.

7. Eisenberg, S., G. Friedman, and T. Vogel. 1988. Enhanced metabolism of normolipidemic human plasma very low density lipoprotein in cultured cells by exogenous apolipoprotein E-3. Arteriosclerosis. 8:480-487.

8. Yamada, N., H. Shimano, H. Mokuno, S. Ishibashi, T. Gotohda, M. Kawakami, Y. Watanabe, Y. Akanuma, T. Murase, and F. Takaku. 1989. Increased clearance of plasma cholesterol after injection of apolipoprotein $E$ into Watanabe heritable hyperlipidemic rabbits. Proc. Natl. Acad. Sci. USA. 86:665-669.

9. Mahley, R. W., K. H. Weisgraber, M. M. Hussain, B. Greenman, M. Fisher, T. Vogel, and M. Gorecki. 1989. Intravenous infusion of apolipoprotein E accelerates clearance of plasma lipoproteins in rabbits. J. Clin. Invest. 83:2125-2130.

10. Mokuno, H., N. Yamada, H. Shimano, S. Ishibashi, N. Mori, K. Takahashi, T. Oka, T. H. Yoon, and F. Takaku. 1991. The enhanced cellular uptake of very low density lipoprotein enriched in apolipoprotein E. Biochim. Biophys. Acta. 1082:63-70.

11. Shimano, H., C. Fukazawa, Y. Shibasaki, N. Mori, T. Gotoda, K. Harada, M. Shimada, N. Yamada, Y. Yazaki, and F. Takaku. 1991. The effect of apo E secretion on lipoprotein uptake in transfected cells. Biochim. Biophys. Acta. 1086:245-254.

12. Shimano, H., N. Yamada, M. Shimada, N. Ohsawa, C. Fukazawa, Y. Yazaki, F. Takaku, and M. Katsuki. 1991. Hepatic and renal expression of rat apolipoprotein $\mathrm{E}$ under control of the metallothionein promotor in transgenic mice. Biochim. Biophys. Acta. 1090:91-94.

13. Shimano, H., N. Yamada, M. Katsuki, M. Shimada, T. Gotoda, K. Harada, T. Murase, C. Fukazawa, F. Takaku, and Y. Yazaki. 1992. Overexpression of apolipoprotein $\mathrm{E}$ in transgenic mice: a marked reduction in plasma lipoproteins except high density lipoprotein, and resistance against diet-induced hypercholesterolemia. Proc. Natl. Acad. Sci. USA. 89:1750-1754.

14. Shimano, H., N. Yamada, M. Katsuki, K. Yamamoto, T. Gotoda, K. Harada, M. Shimada, and Y. Yazaki. 1992. Plasma lipoprotein metabolism in transgenic mice overexpressing apolipoprotein E: accelerated clearance of lipoproteins containing apolipoprotein B. J. Clin. Invest. 90:2084-2091.

15. Havel, R. J., H. A. Eder, and J. H. Bragdon. 1955. The distribution and chemical composition of ultracentrifugally separated lipoproteins in human serum. J. Clin. Invest. 34:1345-1353.

16. McFarlane, A. S. 1958. Efficient trace-labelling of proteins with iodine. Nature (Lond.). 182:53-54.

17. Namba, Y., M. Tomonaga, H. Kawasaki, E. Otomo, and K. Ikeda. 1991 Apolipoprotein $\mathrm{E}$ immunoreactivity in cerebral amyloid deposits and neurofibrillary tangles in Alzheimer's disease and kuru plaque amyloid in Creutzfeldt-Jakob disease. Brain Res. 541:163-166.

18. Laemmli, U. K. 1970. Cleavage of structural proteins during the assembly of the head of bacteriophage T4. Nature (Lond.). 227:680-685.

19. Hamilton, R. L., J. S. Wong, L. S. S. Guo, S. Krisans, and R. J. Havel. 1990. Apolipoprotein E localization in rat hepatocytes by immunogold labeling of cryothin sections. J. Lipid Res. 31:1589-1603.

20. Rubinsztein, D. C., J. C. Cohen, G. M. Berger, D. R. van der Westhuyzen G. A. Coetzee, and W. Gevers. 1990. Chylomicron remnant clearance from the 
plasma is normal in familial hypercholesterolemic homozygotes with defined receptor defects. J. Clin. Invest. 86:1306-1312.

21. Windler, E. E. T., J. Greeve, W. H. Daerr and H. Greten. 1988. Binding of rat chylomicrons and their remnants to the hepatic low-density lipoprotein receptor and its role in remnant removal. Biochem. J. 252:553-561.

22. Choi, S. Y., L. G. Fong, M. J. Kirven, and A. D. Cooper. 1991. Use of an anti-low density lipoprotein receptor antibody to quantify the role of the LDL receptor in the removal of chylomicron remnants in the mouse in vivo. J. Clin. Invest. 88:1173-1181.

23. Pathak, R. K., M. Yokode, R. E. Hammer, S. L. Hoffmann, M. S. Brown, J. L. Goldstein, and R. G. W. Anderson. 1990. Tissue-specific sorting of the human LDL receptor in polarized epithelia of transgenic mice. J. Cell Biol. 111:347-359.

24. Jäckle, S., F. Rinninger, J. Greeve, H. Greten, and E. Windler. 1992. Regulation of the hepatic removal of chylomicron remnants and $\beta$-very low density lipoproteins in the rat. J. Lipid Res. 33:419-429.

25. Windler, E., and R. J. Havel. 1985. Inhibitory effects of $C$ apolipoproteins from rats and humans on the uptake of triglyceride-rich lipoproteins and their remnants by the perfused rat liver. J. Lipid Res. 26:556-565.

26. Kowal, R. C., J. Herz, K. H. Weisgraber, R. W. Mahley, M. S. Brown, and J. L. Goldstein. 1990. Opposing effects of apolipoproteins $E$ and $C$ on lipoprotein binding to low density lipoprotein receptor-related protein. J. Biol. Chem 265:10771-10779.

27. Imaizumi, K., M. Fainaru, and R. J. Havel. 1978. Composition of pro- teins of mesenteric lymph chylomicrons in the rat and alterations produced upon exposure of chyomicrons to blood serum and serum proteins. J. Lipid Res. 19:712-722.

28. Mahley, R. W., and M. M. Hussain. 1991. Chylomicron and chylomicron remnant catabolism. Curr. Opin. Lipidol. 2:170-176.

29. Weisgraber, K. H., S. C. Rall Jr, R. W. Mahley, R. W. Milne, Y. L. Marcel, J. T. Sparrow. 1986. Human apolipoprotein E. Determination of the heparin binding sites of apolipoprotein E3. J. Biol. Chem. 261:2068-2076.

30. Stow, J. L., L. Kellen, E. Unger, M. Hook, and M. G. Farquhar. 1985. Heparan sulfate proteoglycans are concentrated on the sinunoidal plasmalemmal domain and in intracellular organelles of hepatocytes. J. Cell Biol. 100:975-980

31. Ji, Z. S., W. J. Brecht, R. D. Miranda, M. M. Hussain, T. L. Innerarity, and R. W. Mahley. 1993. Role of heparan sulfate proteoglycans in the binding and uptake of apolipoprotein E-enriched remnant lipoproteins by cultured cells. J. Biol. Chem. 268:10160-10167.

32. Mulder, M., P. Lombardi, H. Jansen, T. J. C. van Berkel, R. R. Frants, and L. M. Havekes. 1993. Low density lipoprotein receptor internalizes low density and very low density lipoproteins that are bound to heparan sulfate proteoglycans via lipoprotein lipase. J. Biol. Chem. 268:9369-9375.

33. Mahley, R. W., D. Y. Hui, T. L. Innerarity, and U. Beisiegel. 1989. Chylomicron remnant metabolism-Role of hepatic lipoprotein receptors in mediating uptake. Arteriosclerosis. 9(Suppl.):114-118.

34. Beisiegel, U., W. Weber, and G. Begtsson-Olivecrona. 1991. Lipoprotein lipase enhances the binding of chylomicrons to low density lipoprotein receptorrelated protein. Proc. Natl. Acad. Sci. USA. 88:8342-8346. 